ORIGINAL ARTICLE

\title{
Cardiac complications of enterovirus rhombencephalitis
}

\author{
Y C Fu, C S Chi, Y T Chiu, S L Hsu, B Hwang, S L Jan, P Y Chen, F L Huang, Y Chang
}

Arch Dis Child 2004;89:368-373. doi: 10.1136/adc.2003.029645

See end of article for authors' affiliations .....................

Correspondence to: Dr C S Chi, Department of Pediatrics, Taichung Veterans General Hospital, 160, Section 3 , Chung-Kang Road, Taichung 40705, Taiwan, Republic of China; chi0316@vghtc. vghtc.gov.tw

Accepted 3 August 2003

\begin{abstract}
Background: Epidemics of enterovirus 71 infection have caused the death of many children throughout the world. Rhombencephalitis, brain stem encephalitis, and heart failure were present in all of the fatal cases. However, no evidence of myocarditis was noted in the heart specimens, and the mechanism of heart failure remains unknown.

Aims: To characterise the presentation of cardiac complications in children with enterovirus rhombencephalitis and discuss its pathogenesis.

Methods: Ninety one consecutive patients with enterovirus rhombencephalitis underwent echocardiography. Of these, 17 patients (nine male, eight female; median age 14 months, range 4-57 months) with left ventricular dysfunction were studied.

Results: Tachycardia was noted in all patients and systemic hypertension in 12. Muscle-brain fraction of creatine kinase was $>5 \%$ in 14 patients. Plasma norepinephrine and epinephrine levels were significantly raised in the three patients in whom these were analysed. Electrocardiographic abnormalities were noted in eight patients. Pulmonary oedema was complicated in 15 patients. The initial ejection fraction of the left ventricle was 22-58\% (mean 37\%, SD 11\%). All patients deteriorated to hypotensive shock within 12 hours and 13 died. Heart specimens from seven patients showed no evidence of myocarditis, but significant coagulative myocytolysis, myofibrillar degeneration, and cardiomyocyte apoptosis were observed.

Conclusions: Acute heart failure was noted in 19\% of patients with enterovirus rhombencephalitis, which had a fatality rate of $77 \%$. It was not caused by myocarditis but possibly by neurogenic cardiac damage.
\end{abstract}

$\mathrm{E}$ nteroviral meningoencephalitis generally has a good prognosis, except when the cause is enterovirus $71 .^{1}$ Enterovirus 71 was first isolated in 1969 and has caused epidemics with high mortality rates in many countries, including Bulgaria in 1975 (44 deaths), Hungary in 1978 (47 deaths), Malaysia in 1997 (at least 31 deaths), and Taiwan in 1998 (78 deaths)..$^{2-9}$ According to the Taiwanese Department of Health, the incidence has decreased in recent years but the mortality rate is still high (9 deaths in 1999, 41 deaths in 2000, 58 deaths in 2001). These data indicate that clarification of the cause of death and its pathogenesis are crucial.

Rhombencephalitis, brain stem encephalitis, was the chief neurological complication and was present in all of the fatal cases. ${ }^{6710}$ All patients were fulminate to death in a stereotypical pattern. After experiencing several days of symptoms of hand-foot-mouth disease, herpangina, or febrile illness, patients developed rhombencephalitis. Some developed cardiopulmonary failure and died rapidly despite intensive management. ${ }^{8}$ In the 1998 Taiwan outbreak, Ho et al reported that pulmonary oedema was complicated in most of the patients with fatal outcome (65 of 78 patients) and concluded that most of the patients died of pulmonary oedema. ${ }^{8}$ However, they did not explain the cause of death in the other 13 patients with fatal outcome. In our previous report, among seven patients with fatal outcome, all had left ventricular dysfunction and six had pulmonary oedema. ${ }^{11}$ This finding was similar to that reported by Chan et al in Malaysia. ${ }^{6}$ Of their 24 patients with fatal outcome who had undergone echocardiography and chest radiography, all had left ventricular dysfunction and 17 had pulmonary oedema. ${ }^{6}$ These findings implied that heart failure was the critical cause of rapid death in patients with enterovirus 71 infections. However, there was no evidence of myocarditis in the heart specimens. ${ }^{67}{ }^{11}$ The mechanism of heart failure in these cases remains unknown. The purpose of this study is to characterise the presentation of cardiac complications in children with enterovirus rhombencephalitis and discuss its pathogenesis.

\section{METHODS \\ Patients}

Between April 1998 and June 2002, 91 patients with enterovirus rhombencephalitis were admitted to the paediatric intensive care unit (ICU) of Taichung Veterans General Hospital, a tertiary medical centre in central Taiwan. The diagnosis of rhombencephalitis was based on neurological signs of ataxia, myoclonic jerks, oculomotor problems, or bulbar palsy; or evidence of brain stem involvement from magnetic resonance imaging (MRI) or brain specimen. ${ }^{10}$ The criteria for enterovirus infection were positive culture or reverse transcription polymerase chain reaction (RT-PCR) of enterovirus in specimens of throat swabs. ${ }^{12}$ Since 1999, the enterovirus subtype was further identified by an immunofluorescence assay with enterovirus 71 monoclonal antibody (Chemicon International, Inc., California) and/or positive RTPCR of enterovirus 71..$^{1012}$

After informed consent was obtained, all patients underwent echocardiography. Among them, 17 (nine male, eight female; median age 14 months, range: 4-57 months) with left ventricular dysfunction were studied. None of the patients had a prior history of heart disease. We describe the presentation of heart rate, blood pressure, cardiac enzymes, plasma catecholamines, electrocardiography, chest radiography, echocardiography, cardiac pathology, and outcome.

\section{Virus identification}

Specimens for enterovirus culture were collected in transport medium and inoculated onto monolayers of A549 cells, green monkey kidney cells, and Vero cells within 24 hours. ${ }^{10}$ The cells were inspected daily for a minimum of 14 days for evidence of a viral cytopathic effect. Isolates that produced 


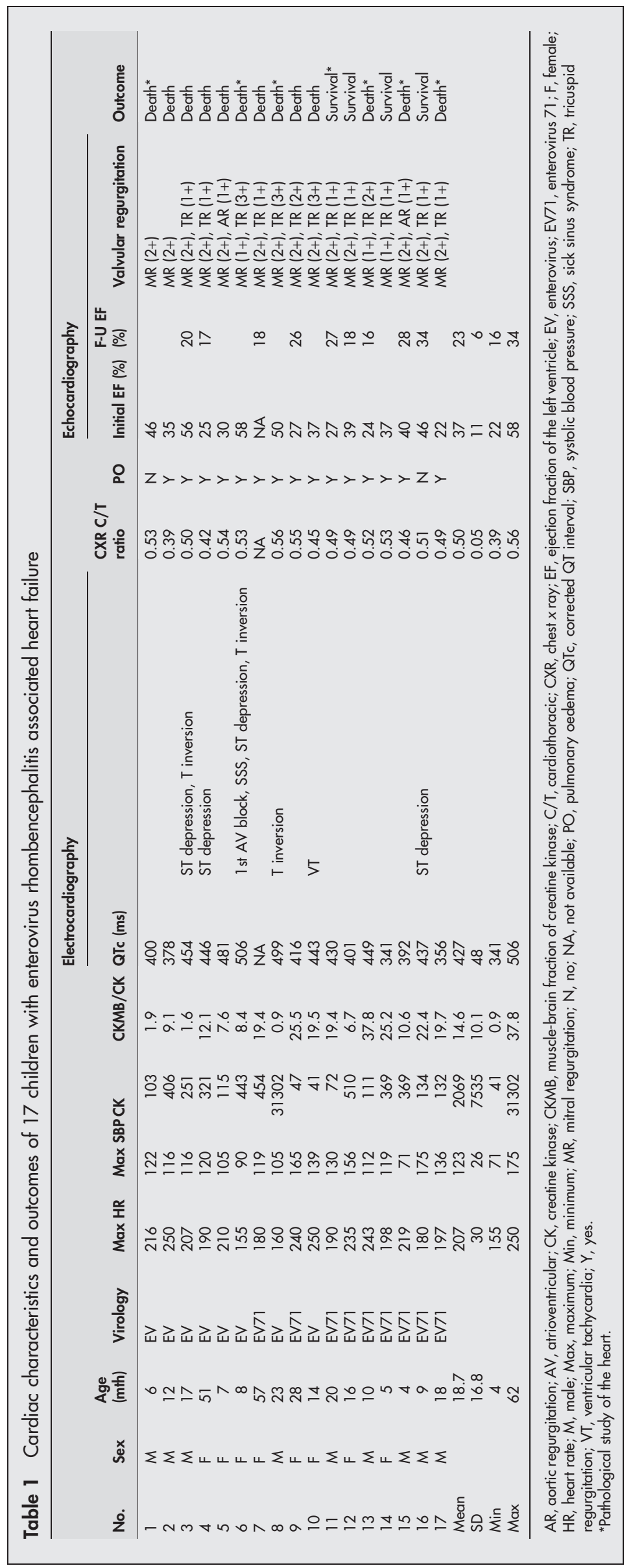




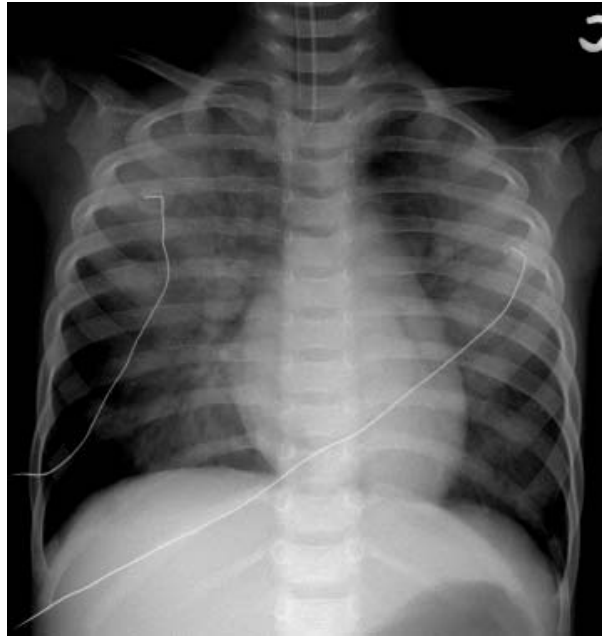

Figure 1 Anteroposterior projection of chest radiography in case 17 shows normal heart size and bilateral pulmonary oedema. At the same time, the ejection fraction of the left ventricle was $22 \%$ measured by echocardiography.

typical enteroviral cytopathic effects were typed by an immunofluorescence assay with enterovirus 71 monoclonal antibodies 3323 and 3324 (Chemicon International, Inc., California). ${ }^{10}$ Isolates that stained for both monoclonal antibodies were identified as enterovirus 71 .

RT-PCR was used to assess enteroviral genome in the specimens of throat swabs and heart tissues. ${ }^{12}{ }^{13}$ Heart tissues were frozen at $-196^{\circ} \mathrm{C}$ and then ground into powder. The specimens from $50 \mathrm{mg}$ heart tissues and throat swabs were assessed using the method of one step RT-PCR assay (Reverse-iT one step kit, ABgene, UK). ${ }^{13}$ Briefly, extracted viral total RNA was converted to CDNA and then PCR amplified in the same tube containing enteroviral primers, RT-PCR master mix (Thermoprime Plus DNA polymerse, optimised reaction buffer, dNTP mix, and $\mathrm{MgCl}_{2}$ ) and reverse-iT Rtase blend reverse transcriptase. Primers used for the common region of enteroviral genome were $3+$ (TCCTCCGGCCCCTGAATG) and $\mathrm{C} \times 10$ (ATTGTGACC ATAAGCAGCCA). ${ }^{12}$ Primers used for specific enterovirus 71 in the VPl specific region were 159S (ACYATGAAAYTGTGCA AGG) and 162A (CCRGTAGGKGTRCACGCRAC)..$^{13}$ The reaction products, 155-bp for non-specific enterovirus and 484-bp for enterovirus 71 , were visualised by ethidium bromide staining under UV transillumination after electrophoretic separation on $2 \%$ agarose gel. A positive and negative control were tested simultaneously.

\section{Heart rate, blood pressure, cardiac enzymes, and plasma catecholamines}

All patients were sedated with midazolam and intubated with mechanical ventilation. Heart rate and blood pressure, measured through the arterial line, were continuously monitored. Maximum heart rate and blood pressure during the period of heart failure were recorded. Serum cardiac enzymes were assayed for creatine kinase (CK) and musclebrain isoenzyme of creatine kinase (CKMB). Plasma was stored at $-70^{\circ} \mathrm{C}$ and the levels of catecholamines including norepinephrine (noradrenaline) and epinephrine (adrenaline) were determined by high performance liquid chromatography. ${ }^{14}$

\section{Electrocardiography and chest radiography}

Twelve lead electrocardiography was performed on admission and read by a paediatric cardiologist. The observed QT

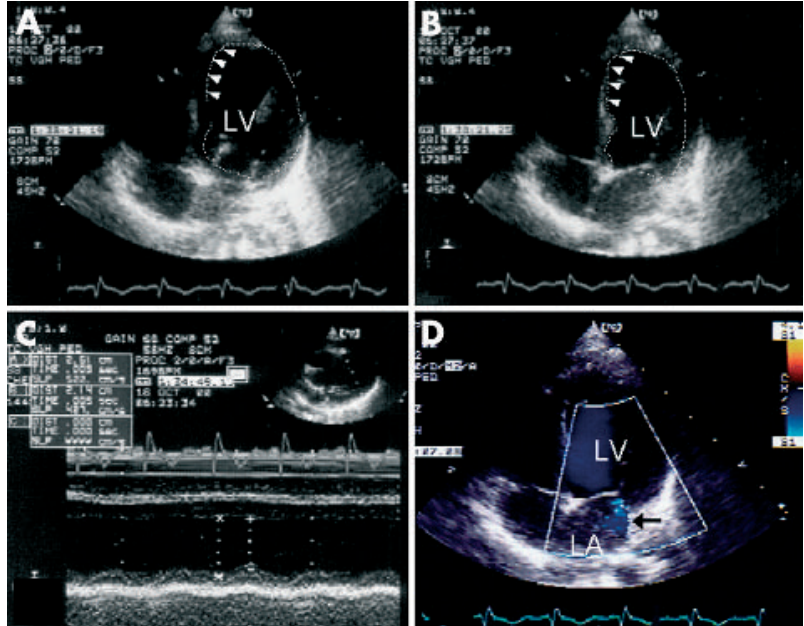

Figure 2 Two dimensional echocardiography in 4-chamber view in case 11 shows generalised hypokinesia of the left ventricle with the ejection fraction of $39 \%$ (panel A in diastole and panel B in systole) Akinesia of apical septum was also noted (arrowheads). M-mode echocardiography shows poor wall motion of the left ventricle (panel C). Colour Doppler echocardiography shows grade 2+ mitral regurgitation (arrow), flowing into the left atrium (panel D). LA, left atrium; LV, left ventricle.

interval was corrected for heart rate (QTc) according to Bazett's formula. ${ }^{15}$ The ST segment elevation or depression was defined as more than $1 \mathrm{~mm}$ of deviation in any lead. Portable chest radiography was undertaken in the supine anteroposterior projection. Cardiothoracic ratio was calculated by relating the largest transverse diameter of the heart to the widest internal diameter of the chest. ${ }^{16}$ Pulmonary oedema was defined as bilateral alveolar infiltrations on the chest radiograph associated with pink foamy tracheal secretion. ${ }^{17}$

\section{Echocardiography}

Transthoracic echocardiography (Hewlett-Packard 5500, USA) was performed within 30 minutes of admission to the ICU and followed up if needed. M-mode echocardiography assessed the ejection fraction of the left ventricle via the parasternal long axis view with a normal range of $64-83 \%{ }^{18}$ Two dimensional echocardiography assessed the wall motion of the left ventricle from the parasternal long axis, short axis, apical 4-chamber, and 2-chamber views. ${ }^{19}$ If regional wall motion abnormality existed, the ejection fraction was measured by biplane Simpson's rule. ${ }^{19}$ Colour Doppler echocardiography assessed valvular regurgitation, which was graded from $1+$ to $4+.^{20}$

\section{Cardiac pathology}

After informed consent was obtained from the patients' parents, heart specimens from seven patients (six postmortem and one biopsy specimens) were sent for pathological study with haematoxylin and eosin stain, Masson's trichrome stain, and in situ terminal deoxyribonucleotidyl transferase mediated dUTP nick end labelling (TUNEL) assay. ${ }^{21}$ All specimens were examined for enteroviral culture and analysed by RT-PCR.

\section{RESULTS}

\section{Patients}

Among the 17 patients with left ventricular dysfunction, 12 (71\%) had hand-foot-mouth disease, two had herpangina, and three had no skin or mucosal lesions. All patients were confirmed to be the victims of enterovirus infection by throat 


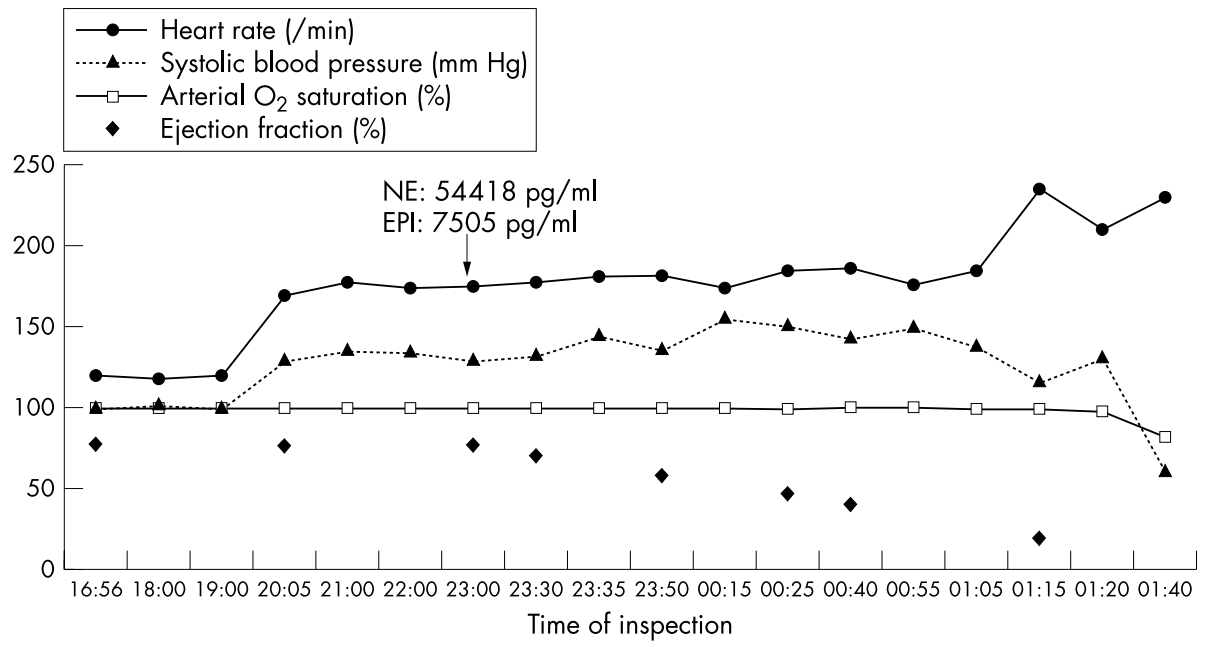

Figure 3 Clinical course in case 12 shows that tachycardia, systemic hypertension, and high concentration of plasma catecholamines preceded the occurrence of heart failure. The arterial oxygen saturation was around 100\% throughout the course. The patient eventually deteriorated to hypotensive shock.
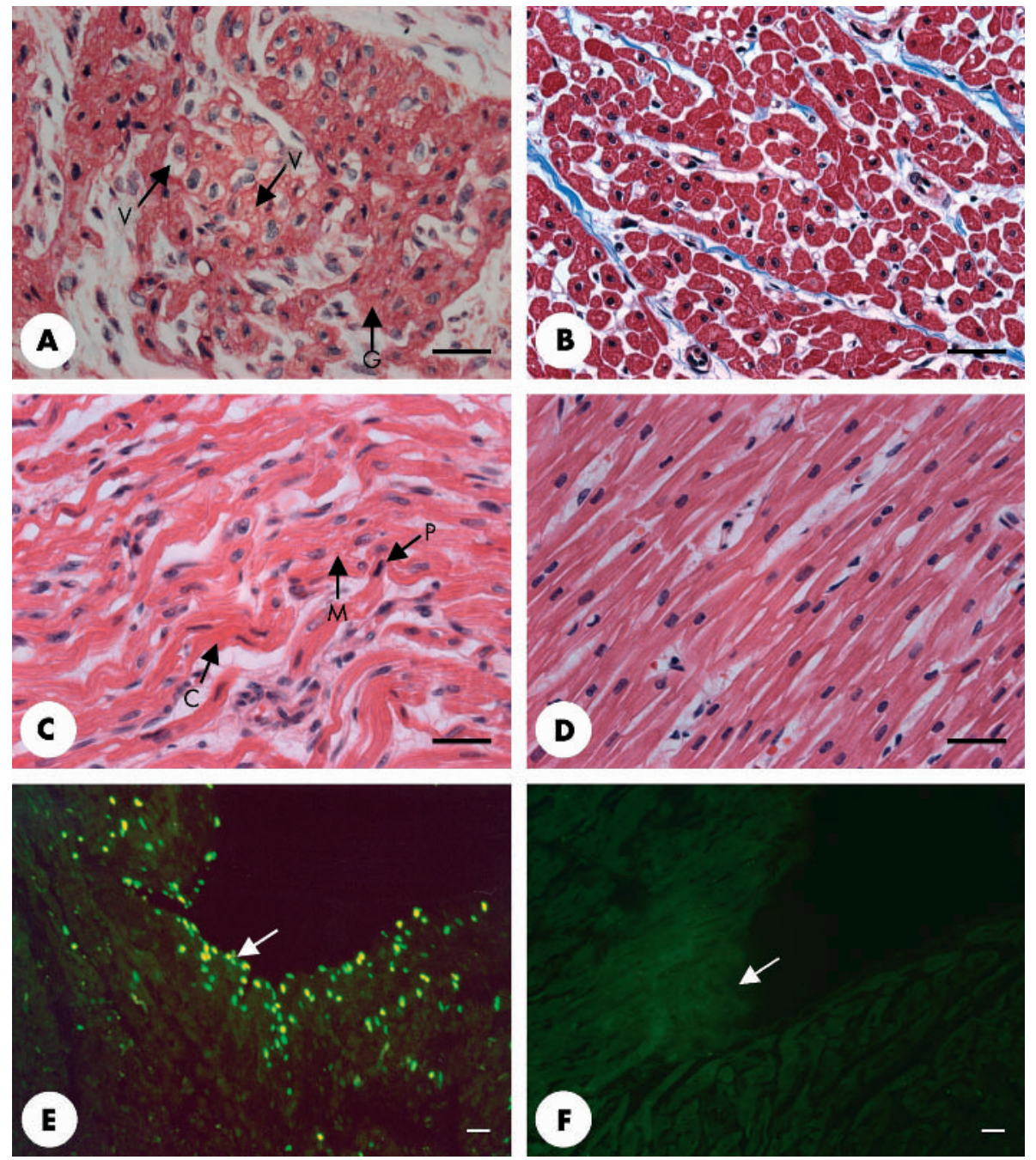

Figure 4 Histopathological examinations of the heart specimens from study patients (left panels) were compared to those from a control, a 4 year old boy who died of sepsis (right panels). Panel A in Masson's trichrome stain and panel C in haematoxylin and eosin stain show significant coagulative myocytolysis and myofibrillar degeneration and waving in the left ventricle, indicated by sarcoplasmic coagulation (C), granulation (G), vacuolisation (V), and myocytolysis (M). Some nuclei became condensed, pyknosis (P), and irregular in shape. Panel E shows significant cardiomyocyte apoptosis, indicated by green fluorescent nuclei and was more remarkable in the subendocardial region (arrow) (in situ TUNEL assay). The bar scales are $30 \mu \mathrm{m}$ in length. 
culture in 13 and RT-PCR in 15. All nine cases after 1999 were further identified to be infected by enterovirus 71 . Neurological symptoms included myoclonic jerks in 12 patients, opsoclonus in five, abducent nerve palsy in three, acute flaccid paralysis in three, and ataxia in three. All four survivors underwent brain MRI, which showed high signal lesions in the pons and medulla in all and cervical spinal cord involvement in one patient.

\section{Heart rate, blood pressure, cardiac enzymes, and plasma catecholamines}

Table 1 summarises the cardiac characteristics and outcome of patients. All patients had tachycardia with a maximum heart rate of 155-250/min (mean (SD) 207 (30)/min), and 12 had systemic hypertension >115 mm Hg despite the coexistence of heart failure. Fourteen patients exhibited CKMB/ CK ratio $>5 \%$. In three patients (cases 12, 13, and 15) plasma catecholamines were measured; results showed norepinephrine levels of 4723, 54418, and 579 pg/ml (normal range 100$400 \mathrm{pg} / \mathrm{ml}$ ) and epinephrine levels of 3461, 7505, and $6137 \mathrm{pg} / \mathrm{ml}$ (normal range $<70 \mathrm{pg} / \mathrm{ml}$ ), respectively.

\section{Electrocardiography and chest radiography}

Eight $(47 \%)$ patients had electrocardiographic abnormalities, including prolonged QT interval in seven, ST depression in four, $\mathrm{T}$ inversion in three, ventricular tachycardia in one, and first degree atrioventricular block with sick sinus syndrome in one. Fourteen $(88 \%)$ patients had normal heart size on chest radiography with the cardiothoracic ratio $<0.55$ (fig l). Pulmonary oedema was complicated in 15 patients.

\section{Echocardiography}

The initial abnormal ejection fraction of the left ventricle was $22-58 \%$ (mean (SD) $37 \%(11 \%)$ ) and follow up ejection fraction was $16-34 \%$ (mean (SD) 23\% (6\%)) (table 1). Two dimensional echocardiography showed generalised hypokinesia of the left ventricle in all patients, in three combined with akinesia of the apical septum and in two with akinesia of the basal septum (fig 2). Case 6 was associated with significant right ventricular dysfunction and dilatation. Colour Doppler echocardiography showed mitral regurgitation in all patients and tricuspid regurgitation $\geqslant$ grade $2+$ in five and mild aortic regurgitation in two.

\section{Clinical course}

All patients deteriorated to hypotensive shock within 12 hours after detection of the heart failure. Thirteen patients (77\%) died despite aggressive cardiopulmonary management. Figure 3 represents the clinical course of case 12, which shows the tachycardia, systemic hypertension, and high concentration of plasma catecholamines preceding the occurrence of heart failure. However, the arterial oxygen saturation was around 100\% throughout the course. The patient eventually deteriorated to hypotensive shock.

\section{Cardiac pathology}

Pathological investigation of seven patients demonstrated that all ventricular specimens showed significant coagulative myocytolysis and myofibrillar degeneration, which were indicated by sarcoplasmic coagulation, granulation, and vacuolisation (fig $4 \mathrm{~A}$ and $4 \mathrm{C}$ ). A significant portion of the nuclei became condensed (pyknosis) and irregular in shape (fig 4C). Myofibrillar waving and lysis were observed (fig 4C). In situ TUNEL assay showed various degrees of cardiomyocyte apoptosis, which was more remarkable in the subendocardial region (fig 4E). Examination of all specimens showed that the left ventricle was more remarkably involved than the right ventricle except in case 6. Atrial specimens showed normal to mild myocytolysis. None of the heart specimens showed evidence of a viral presence from culture or RT-PCR, or the infiltration of inflammatory cells.

\section{DISCUSSION}

Enterovirus 71 is a neurotropic virus, which can cause aseptic meningitis, encephalitis, acute flaccid paralysis, or rhombencephalitis. ${ }^{10}$ Huang et al confirmed that rhombencephalitis was the chief neurological syndrome in the 1998 Taiwan outbreak and responsible for all the fatal cases. ${ }^{10}$ The lesion was most commonly in the pontine tegmentum with subsequent rostral and caudal progression, involving the vasomotor centre. ${ }^{10}$ The mortality rate was $14 \%$ (5/37) in patients with rhombencephalitis. ${ }^{10}$ All of the patients with fatal outcome presented with rapid cardiopulmonary failure and died within 12 hours after admission to the hospital despite receiving cardiopulmonary support. ${ }^{10}$ Nevertheless, Huang et al did not clarify the role of the heart in these fatal cases.

Our data revealed that characteristic presentation of cardiac complications in patients with enterovirus 71 rhombencephalitis included the following. First, it was not caused by myocarditis. Second, hypersympathetic activity, including tachycardia, systemic hypertension, and high levels of plasma catecholamines, preceded or coexisted with the left ventricular dysfunction. Third, cardiac enzymes were increased in most patients. Fourth, electrocardiographic abnormalities occurred about half the patients. Fifth, normal heart size on chest radiography was noted in most patients. Sixth, it was usually associated with pulmonary oedema. Seventh, left ventricular dysfunction was acute and usually fatal. Eighth, coagulative myocytolysis, myofibrillar degeneration, and cardiomyocyte apoptosis were noted in all ventricular specimens, which were remarkable in the subendocardial region of the left ventricle.

All of the above manifestations are similar to those in patients with neurogenic cardiac damage. ${ }^{22-26}$ Neurogenic cardiac damage has been studied for decades and has been observed in patients with subarachnoid haemorrhage, head trauma, status epilepticus, and stroke. ${ }^{22-26}$ Sato et al reported that transient left ventricular asynergy was noted in $9.4 \%$ $(67 / 715)$ of patients with subarachnoid haemorrhage. ${ }^{23}$ Plasma catecholamine concentrations were higher in these patients compared with those without left ventricular asynergy. ${ }^{23}$ Pollick et al performed a prospective two dimensional echocardiographic study and found that all patients had akinesia of the apical septum initially and some of them progressed to include additional wall motion abnormalities. ${ }^{26}$ Marion et al reported that $50-72 \%$ of patients with subarachnoid haemorrhage had electrocardiographic abnormalities including $\mathrm{T}$ wave abnormalities in $47 \%$ of patients, QT prolongation in $37 \%$, rhythm abnormalities in $35 \%$, ST segment changes in $30 \%$, and prominent $\mathrm{U}$ waves in $25 \%{ }^{24}$ The consensus pathological findings of neurogenic cardiac damage are coagulative myocytolysis and myofibrillar degeneration, which are greatest near the nerve terminals in the endocardium. ${ }^{22} 27$ They are also known as contraction band necrosis, in which the cells die in a hypercontracted state with a prominent contraction band. ${ }^{22} 27$ These lesions are the characteristics of catecholamine cardiotoxicity and are also noted in patients with pheochromocytoma. ${ }^{27}$

The mechanism of neurogenic cardiac damage is generally believed to result from excessive catecholamine release caused by a brain lesion. ${ }^{22-26}$ Based on these observations, we propose the following mechanism of cardiac damage in patients with enterovirus 71 rhombencephalitis. The neurotropic enterovirus 71 infects the brain stem (rhombencephalitis), resulting in damage to the dorsal vagus nuclei and nuclei of the solitary tract. Subsequent inactivation of the parasympathetic system and activation of the nearby central 
sympathetic pathway cause a "catecholamine storm", which results in catecholamine cardiotoxicity including coagulative myocytolysis, myofibrillar degeneration, and cardiomyocytes apoptosis.

Apoptosis is programmed cell death that does not induce inflammatory reaction. ${ }^{28}$ It is an important mechanism to produce heart failure. ${ }^{28}{ }^{29}$ According to many in vitro and in vivo studies, ${ }^{28-30}$ excessive catecholamines are able to induce the apoptosis of cardiomyocytes. Our study reveals that apoptosis plays a role in the pathogenesis of heart failure in patients with enterovirus 71 rhombencephalitis. To our knowledge, this is the first report that shows the role of apoptosis in neurogenic cardiac damage. However, further study is required to clarify the precise mechanism.

The heart failure observed in patients with enterovirus 71 rhombencephalitis is more severe than that observed in patients with subarachnoid haemorrhage. Our explanation is that subarachnoid haemorrhage activates the sympathetic centre (hypothalamus), while enterovirus rhombencephalitis not only activates the central sympathetic pathway but also suppresses parasympathetic activity. More intense and longer catecholamine release is induced in the latter case.

Our data showed that $88 \%$ of patients exhibited normal heart size on the chest radiograph and the heart failure coexisted with normotension or hypertension, which usually cause a clinician to overlook the existence of cardiac dysfunction. The implications for treatment include the following. Echocardiography should be performed as soon as possible and followed up frequently in patients with enterovirus 71 rhombencephalitis. If cardiac dysfunction is found, fluid restriction and cautious use of a vasodilator are suggested. Inotropic agents derived from catecholamines should be avoided, while those derived from phosphodiesterase inhibitors, such as milrinone, might be considered. If cardiac function deteriorates despite medical treatment, extracorporeal circulatory life support might be indicated. ${ }^{31}$ Based on the proposed mechanism, a sympathetic blocking agent may be beneficial in the early stages to prevent catecholamine toxicity but this demands further study before clinical implementation.

In conclusion, acute heart failure was noted in $19 \%$ of patients with enterovirus rhombencephalitis, which had a fatality rate of $77 \%$. It was not caused by myocarditis but possibly by neurogenic cardiac damage.

\section{ACKNOWLEDGEMENTS}

We are indebted to Chi-Ren Tsai and Li-Cheng Wang for their identification of enterovirus; to Yu-Ping Wang for her assistance in performing the echocardiography; to Sui-Chu Yin for histological staining; and to Professor Shuu-Jiun Wang, National Yang-Ming University, for his valuable suggestions.

\section{Authors' affiliations}

Y C Fu, C S Chi, S L Jan, P Y Chen, F L Huang, Departments of Pediatrics, Taichung Veterans General Hospital, Taichung, Taiwan

Y T Chiu, S L Hsu, Department of Medical Research, Taichung Veterans General Hospital, Taichung, Taiwan

Y Chang, Section of Cardiovascular Surgery, Department of Surgery,

Taichung Veterans General Hospital, Taichung, Taiwan

B Hwang, Institute of Clinical Medicine, National Yang-Ming University, Taipei, Taiwan

\section{REFERENCES}

1 Cherry JD. Enteroviruses: coxsackieviruses, echoviruses, and polioviruses. In: Feigin RD, Cherry JD, eds. Textbook of pediatric infectious diseases, 4th edn. Philadelphia: WB Saunders, 1998:1787-839

2 Schmidt NJ, Lennette EH, Ho HH. An apparently new enterovirus isolated from patients with disease of the central nervous system. J Infect Dis 1974:129:304-9.

3 Shindarov LM, Chumakov MP, Voroshilova MK, et al. Epidemiological, clinical, and pathomorphological characteristics of epidemic poliomyelitis-like disease caused by enterovirus 71. J Hyg Epidemiol Microbiol Immunol 1979;23:284-95.

4 Nagy G, Takatsy S, Kukan E, et al. Virological diagnosis of enterovirus type 71 infections: experiences gained during an epidemic of acute CNS diseases in Hungary in 1978. Arch Virol 1982;71:217-27.

5 World Health Organisation. Outbreak of hand, foot and mouth disease in Sarawak. Cluster of deaths among infants and young children. Wkly Epidemiol Rec 1997;72:211-12.

6 Chan LG, Parashar UD, Lye MS, et al. Deaths of children during an outbreak of hand, foot, and mouth disease in Sarawak, Malaysia: clinical and pathological characteristics of the disease. Clin Infect Dis 2000;31:678-83.

7 Lum LCS, Wong KT, Lam SK, et al. Fatal enterovirus 71 encephalomyelitis. J Pediatr 1998;133:795-8.

8 Ho $M$, Chen ER, Hsu KH, et al. An epidemic of enterovirus 71 infection in Taiwan. N Engl J Med 1999;341:929-35.

9 Deaths among children during an outbreak of hand, foot, and mouth disease-Taiwan, Republic of China, April-July 1998. MMWR Morb Mortal Wkly Rep 1998;47:629-32 [Erratum, MMWR Morb Mortal Wkly Rep 1998;47:718].

10 Huang CC, Liu CC, Chang YC, et al. Neurologic complications in children with enterovirus 71 infection. N Engl J Med 1999;341:936-42.

11 Jan SL, Chi CS, Hwang B, et al. Cardiac manifestations of fatal enterovirus infection during the 1998 outbreak in Taiwan. Chung Hua I Hsueh Tsa Chih (Taipei) 2000;63:612-18.

12 Zoll GJ, Melchers WJ, Kopecka H, et al. General primer-mediated polymerase chain reaction for detection of enteroviruses: application for diagnostic routine and persistent infections. J Clin Microbiol 1992;30:160-5.

13 Brown BA, Kilpatrick DR, Oberste MS, et al. Serotype-specific identification of enterovirus 71 by PCR. J Clin Virol 2000;16:107-12.

14 Cheng FC, Yang LL, Chang FM, et al. Simultaneous measurement of serotonin, catecholamines and their metabolites in cat and human plasma by in vitro microdialysis-microbore high-performance liquid chromatography with amperometric detection. J Chromatogr 1992;582:19-27.

15 Bazett HC. An analysis of the time relations of electrocardiograms. Heart 1920;7:353-67.

16 Satou GM, Lacro RV, Chung T, et al. Heart size on chest $x$-ray as a predictor of cardiac enlargement by echocardiography in children. Pediatr Cardiol 2001;22:218-22.

17 Colice GL. Detecting the presence and cause of pulmonary edema. Postgrad Med 1993;93:161-6, 169-70.

18 Park MK. Pediatric cardiology for practitioners, 3rd edn. St Louis: MosbyYear Books, 1996:67-82.

19 Silverman NH. Pediatric echocardiography. Baltimore: Williams \& Wilkins, 1993:35-108.

20 Miyatake K, Izumi S, Okamoto M, et al. Semiquantitative grading of severity of mitral regurgitation by real-time two-dimensional Doppler flow imaging technique. J Am Coll Cardiol 1986;7:82-8.

21 Heatwole VM. TUNEL assay for apoptotic cells. Methods Mol Biol 1999;115:141-8.

22 Samuels MA. Neurally induced cardiac damage. Definition of the problem. Neurol Clin 1993;11:273-92.

23 Sato K, Masuda T, Izumi T. Subarachnoid hemorrhage and myocardial damage: clinical and experimental studies. Jpn Heart J 1999:40:683-701.

24 Marion DW, Segal R, Thompson ME. Subarachnoid hemorrhage and the heart. Neurosurgery 1986;18:101-6.

25 Connor RC. Heart damage associated with intracranial lesions. BMJ 1968;3:29-31.

26 Pollick C, Cujec B, Parker S, et al. Left ventricular wall motion abnormalities in subarachnoid hemorrhage: an echocardiographic study. J Am Coll Cardiol 1988; 12:600-5.

27 Baroldi G. Myocardial cell death, including ischemic heart disease and its complications. In: Silver MD, Gotlieb Al, Schoen FJ, eds. Cardiovascular pathology, 3rd edn. Philadelphia: Churchill Livingstone, 2001:202-6.

28 Williams RS. Apoptosis and heart failure. N Engl J Med 1999;341:759-60.

29 Narula J, Haijar RJ, Dec GW. Apoptosis in the failing heart. Cardiol Clin 1998;16:691-710

30 Communal C, Singh K, Pimentel DR, et al. Norepinephrine stimulates apoptosis in adult rat ventricular myocytes by activation of the beta-adrenergic pathway. Circulation 1998;98:1329-34.

31 Huang $\mathrm{FL}$, Jan SL, Chen PY, et al. Left ventricular dysfunction in children with fulminant enterovirus 71 infection: an evaluation of the clinical course. Clin Infect Dis 2002;34:1020-4. 